\title{
Intra-osseous Co-transplantation of CD34-selected Umbilical Cord Blood and Mesenchymal Stromal Cells
}

\author{
Leland Metheny III',2*, Saada Eid ${ }^{3}$, Karen Lingas ${ }^{2}$, Jane Reese $^{2}$, Howard Meyerson ${ }^{4}$, Alexander Tong ${ }^{2}$, Marcos de Lima ${ }^{1,2}$ and Alex Y. Huang $^{3,4}$ \\ ${ }^{1}$ Stem Cell Transplant Program, University Hospitals Cleveland Medical Center and Case Western Reserve University, Cleveland, OH 44106, USA \\ ${ }^{2}$ Case Comprehensive Cancer Center, Case Western Reserve University School of Medicine, Cleveland, OH 44106, USA \\ ${ }^{3}$ Division of Pediatric Hematology/Oncology, Department of Pediatrics, Case Western Reserve University School of Medicine; Angie Fowler AYA Cancer Institute, \\ University Hospitals Rainbow Babies \& Children's Hospital, Cleveland, OH 44106, USA \\ ${ }^{4}$ Department of Pathology, Case Western Reserve University School of Medicine; University Hospitals Cleveland Medical Center, Cleveland, OH 44106, USA
}

\begin{abstract}
Human mesenchymal stromal cells (MSC) have been shown to support the growth and differentiation of hematopoietic stem cells (HSC). We hypothesized that intra-osseous (IO) co-transplantation of MSC and umbilical cord blood (UCB) may be effective in improving early HSC engraftment, as IO transplantation has been demonstrated to enhance UCB engraftment in NOD SCID-gamma (NSG) mice. Following non-lethal irradiation (300rads), 6 groups of NSG mice were studied: 1) intravenous (IV) UCB CD34+ cells, 2) IV UCB CD34+ cells and MSC, 3) IO UCB CD34 cells, 4) IO UCB CD34+ cells and IO MSC, 5) IO UCB CD34+ cells and IV MSC, and 6) IV UCB CD34 $4^{+}$and IO MSC. Analysis of human-derived CD45 ${ }^{+}, \mathrm{CD} 3^{+}$, and $\mathrm{CD} 19^{+}$cells 6 weeks following transplant revealed the highest level of engraftment in the IO UCB plus IO MSC cohort. Bone marrow analysis of human CD13 and CD14 markers revealed no significant difference between cohorts. We observed that IO MSC and UCB co-transplantation led to superior engraftment of CD45+, CD3 $3^{+}$and $\mathrm{CD} 19^{+}$lineage cells in the bone marrow at 6 weeks as compared with the IV UCB cohort controls. Our data suggests that IO co-transplantation of MSC and UCB facilitates human HSC engraftment in NSG mice.
\end{abstract}

\section{Abbreviations and symbols}

Human mesenchymal stromal cells (MSC); Hematopoietic stem cells (HSC); Umbilical cord blood (UCB); Intra-osseous (IO); Intravenous (IV); Non-obese diabetic severe combined immunodeficiency- IL2Rgammanull (NSG); Stem cell transplant (SCT); Monoclonal antibodies (mAbs); Phosphate Buffered Saline (PBS); Human Serum Albumin (HSA); Institutional Animal Care and Use Committee (IACUC); Standard error of the mean (SEM)

\section{Introduction}

Umbilical cord blood (UCB) is rich in hematopoietic stem cells (HSC) and it has been used as a graft source for both hematopoietic stem cell transplant (SCT) patients and murine models of transplant $[1,2]$. Usually, UCB is given after conditioning chemotherapy or radiation through intravenous (IV) infusion. However, due to delayed or sub-optimal engraftment in both mice and humans, other methods beyond IV infusion have been proposed. These include intra-osseous infusion, co-infusion of the UCB with mesenchymal stromal cells (MSC), or ex vivo expansion of UCB prior to infusion [3-5]. Here we report our data on intra-osseous co-infusion of UCB and MSC in a murine model of transplant.

It has been shown that a large percentage of HSCs, when introduced IV, do not reach the bone marrow niche [6,7]. Cui et al. demonstrated that when fluorescently labelled donor bone marrow cells are injected IV in an irradiated syngeneic mouse model, only 1-2\% of donor HSCs reached the bone marrow [6]. In mouse models, direct IO injection of HSC improves overall engraftment, possibly by bypassing potential "trapping" sites in multiple organs including the lung, liver, spleen and kidney [8-10]. In an experiment performed by Castello et al. direct IO inoculation of UCB resulted in higher engraftment rates when compared to IV UCB injection 30 days post transplantation, as measured by the abundance of human myeloid cells, identified by $\mathrm{CD} 45^{+}$cells within the bone marrow [9].

The bone marrow microenvironment provides an important niche for the proliferation and differentiation of HSC [11-14]. Human MSC have been shown to support the growth and differentiation of HSC [15]. Bone marrow MSC support hematopoietic growth through a number of mechanisms including the production of IL-6, IL-11, leukemia inhibitory factor, stem cell factor and thrombopoietin [16,17]. MSC also aid in the homing and migration of HSC to the bone marrow [18]. Furthermore, the addition of MSC ex vivo to UCB cultures results in significant expansion of the hematopoietic stem $\left(\mathrm{CD} 34^{+}\right)$cell pool. This method of ex vivo expansion has been used to increase the cell dosages of UCB grafts in patients undergoing SCT [12].

MSCs have also been co-infused with CD34-selected human UCB IV in a murine model of hematopoietic stem cell transplant [19]. In this model, mice transplanted with CD34 selected UCB and Stro-1- MSCs exhibited significantly greater human hematopoietic engraftment in the

Correspondence to: Leland Metheny, Stem Cell Transplant Program, University Hospitals Case Medical Center and Case Western Reserve University, Cleveland, OH 44106, USA, Tel: 216-844-0139; Fax: 216-201-5451; E-mail: Leland. metheny@uhhospitals.org

Key words: cord blood stem cell transplantatio, mesenchymal stromal cells, hematopoietic stem cell transplantation

Received: October 11, 2016; Accepted: October 18, 2016; Published: October 20,2016 
bone marrow, spleen and blood (CD45\%: 48, 35, and 14, respectively) when compared to mice transplanted with UCB alone (CD45\%: 27, 15, and 4 , respectively) at 12 weeks following transplantation. These data formed the basis for the approach of using a combination of MSC and UCB co-transplantation IV in clinical trials, which were found to be safe [20].

Carrancio et al. demonstrated that in a mouse transplantation model, co-infusion of human MSCs and human CD34 ${ }^{+} \mathrm{UBC}$ enhanced the engraftment of myeloid compartment when compared to controls [21]. Mice co-transplanted with MSC and UCB by either the IV or IO route had greater B-cell $\left(\mathrm{CD} 19^{+}\right)$and myeloid $\left(\mathrm{CD} 13^{+}\right)$ chimerism at 3 weeks following transplantation. Furthermore, mice transplanted with IO UCB and IO MSC had the greatest rate of human engraftment $\left(\mathrm{CD} 45^{+}\right)$in the injected femur. Based on the data above, we hypothesized that co-transplantation of MSC and UCB via direct IO route would further improve early human engraftment in a mouse transplantation model.

\section{Methods}

\section{Mice}

Non-Obese Diabetic- Severe Combined ImmunodeficiencyIL2Rgammanull (NSG) mice were from breeding pairs originally purchased from Jackson Laboratories. NSG were bred in a pathogenfree unit and maintained in sterile cages.

\section{Human MSCs}

Human MSC $\left(\mathrm{CD} 105^{+} \mathrm{CD}^{+} 3^{+} \mathrm{CD} 45^{-} \mathrm{CD} 14^{-}\right)$were obtained from bone marrow donors using Percoll gradient isolation and cultureexpanded to a homogeneous population under approved protocols in the National Center for Regenerative Medicine/Seidman Cancer Center Cellular Therapy Lab. MSCs were preserved in DMSO and when needed were thawed and re-suspended at the appropriate concentration per cohort.

\section{$\mathrm{CD}^{+}{ }^{+} \mathrm{UCB}$ cell isolation}

Cord blood units were received from the Cleveland Cord Blood Center. Each unit was diluted 1:3 with Phosphate Buffered Saline (PBS) $+0.5 \%$ Human Serum Albumin (HSA) and layered onto Ficoll Paque PLUS to isolate the mononuclear cells by density gradient. After a cell count and washes, the mononuclear cells were labeled per protocol using the Miltenyi Biotec CD34 Microbead Kit. The CD34 cells were then isolated using an LS column in a magnet and washed three times with MACS buffer. The CD34 cells from both cord blood units were combined, counted, and re-suspended in PBS for injection into mice.

\section{Murine transplantation}

Female Nude Obese Diabetic Severe Combined Immunodeficiency (NSG) mice aged 8 to 12 weeks were purchased from Jackson Laboratory (Bar Harbor, ME). Prior to SCT, recipient mice were ear punched for individual identification. Mice received allogeneic SCT using CD34 selected human umbilical cord blood. NSG mice received 300 cGy TBI immediately prior to receiving $5 \times 10^{5} \mathrm{CD} 34^{+}$selected cells from human umbilical cord blood with or without $1 \times 10^{6}$ human MSC.

Six groups of NSG mice were studied: 1) intravenous (IV) $5 \times 10^{5}$ $\mathrm{UCB} \mathrm{CD} 4^{+}$cells, 2) IV $5 \times 10^{5} \mathrm{UCB} \mathrm{CD} 34^{+}$cells and $1 \times 10^{6} \mathrm{MSC}$, 3) IO $5 \times 10^{5} \mathrm{UCB} \mathrm{CD} 34^{+}$cells, 4) IO $5 \times 10^{5} \mathrm{UCB} \mathrm{CD} 34+$ cells and IO $1 \times 10^{6} \mathrm{MSC}$, 5) IO $5 \times 10^{5} \mathrm{UCB} \mathrm{CD} 34^{+}$cells and IV $1 \times 10^{6} \mathrm{MSC}$, and 6) IV $5 \times 10^{5}$ UCB CD34+ and IO $1 \times 10^{6}$ MSC. MSC dose was arbitrarily set at 2:1 with UCB CD34+ cell dose. IV injections: cells were administered via tail vein injection suspended in a total volume of 200 $\mu l$. IO injections: Mice were anesthetized using fluorine gas, the left leg was shaved, the hair removed and betadine was applied to the skin. Cells were administered via bilateral tibia IO injections suspended in a total volume of $40 \mu \mathrm{l}(20 \mu \mathrm{l}$ in each tibia) using a 30-gauge needle. There were five mice in each cohort. Flow Cytometry: Peripheral blood samples from mice were analyzed on weeks 2, 4 and 6. Bone marrow tissue samples from mice were analyzed on week 6 . Peripheral blood and bone marrow samples were stained for T cell markers, (CD3), myeloid markers (CD45, CD13, CD14) and with B-cell markers (CD19). Specific lineage markers (CD3, CD13, CD14 and CD19) were analyzed inside the human $\mathrm{CD} 45^{+}$population. All monoclonal antibodies (mAbs) were purchased from BD Biosciences Pharmingen (San Diego, CA) or eBioscience. At least $1 \times 10^{5}$ events were analyzed per conjugated MAb stain condition. Data were analyzed using CFlow.

The liver, ileum, ascending colon, and right tibia were harvested. Organs were fixed in $10 \%$ buffered formalin, embedded in paraffin, cut into $5 \mu \mathrm{m}$-thicksections, and stained with hematoxylin and eosin for histological examination. Slides were coded without reference to transplant group or treatment and reviewed in blinded fashion by a single pathologist. All animal studies were approved by the Institutional Animal Care and Use Committee (IACUC) at Case Western Reserve University (IACUC protocol 2015-0118).

\section{Histology}

An independent hematopathologist, blinded to cohort characteristics, evaluated tibial sections, one from each mouse in every cohort (for a total of 5 per cohort), for myeloid-to-erythroid ratio, cellularity, and megakaryocyte percentages.

\section{Statistics}

All values are expressed as the mean plus or minus standard error of the mean $( \pm$ SEM). Statistical comparisons between groups were completed using Mann and Whitney test (nonparametric data).

\section{Results}

\section{IO co-transplantation of $\mathrm{CD} 34^{+} \mathrm{UCB}$ and MSC improves overall HSC engraftment and enhances $\mathrm{CD3}^{+} \mathrm{T}$ cell and $\mathrm{CD}^{+} 9^{+} \mathrm{B}$ cell recovery}

Following irradiation, recipient mice were injected with MSC and $\mathrm{CD} 34^{+} \mathrm{UCB}$ via either the IV or IO route. At six weeks following transplantation, we assessed the degree of hematopoietic engraftment that can be traced to human $\mathrm{CD}^{+} 4^{+}$lineage by measuring cells that stained positive for human CD45 by flow cytometry. Cellular engraftment was significantly enhanced in the group receiving the IO co-transplantation of UCB and MSC as compared to IV UCB alone ( $34 \%$ vs. $2.9 \%$, respectively; Figure 1 and Table $1 ; \mathrm{p}<0.001$ ). Consistent with our hypothesis that administration of MSC improves overall UCB engraftment, we observed that any combination of UCB and MSC coadministration, irrespective of the mode of delivery of either the UCB or MSC, exhibited significantly improved HSC engraftment as compared to IV UCB injection alone. In particular, direct IO administration of UCB in the presence of either IO or IV MSC led to a trend towards improved engraftment at 6 weeks among $\mathrm{CD} 45^{+}$lineage cells as well as $\mathrm{CD}^{+}, \mathrm{CD}^{+} 3^{+}, \mathrm{CD} 14^{+}$and $\mathrm{CD} 19^{+}$cells (Table 1$)$.

$\mathrm{CD}^{2} 9^{+} \mathrm{B}$ cell and $\mathrm{CD}^{+} \mathrm{T}$ cells recovery was significantly increased ( $\mathrm{p}<0.001$ and $\mathrm{p}<0.05$, respectively) in the IO UCB and MSC co- 


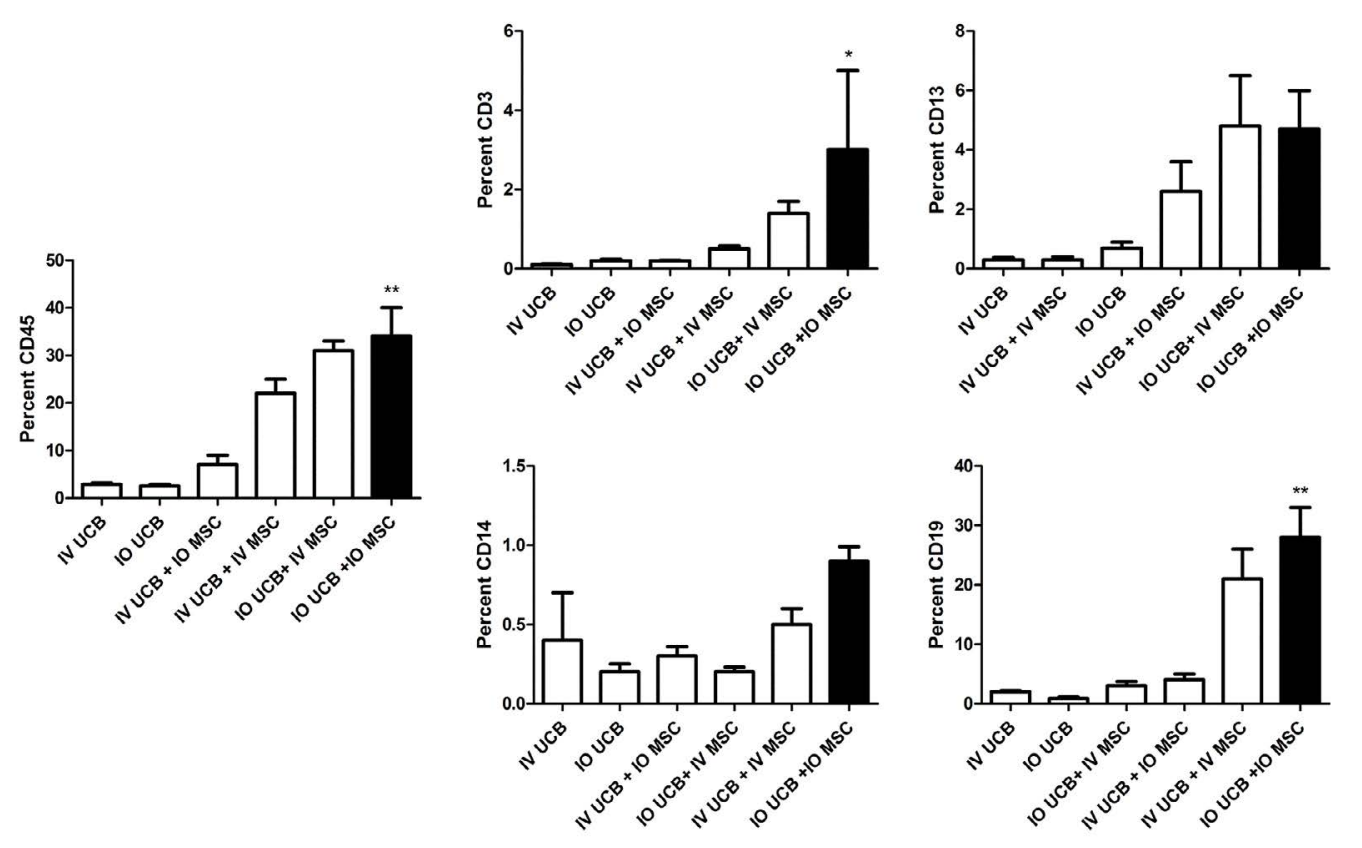

Figure 1. Percent CD45, CD3, CD13, CD14, CD19 in the right tibia bone marrow at 6 weeks post umbilical cord blood infusion. IV UCB $=$ intravenous umbilical cord blood, IO UCB $=$ intra-osseous umbilical cord blood, IV UCB + IV MSC = intravenous umbilical cord blood and mesenchymal stromal cells, IO UCB + IO MSC $=$ intra-osseous umbilical cord blood and mesenchymal stromal cells, IV UCB + IO MSC = intravenous umbilical cord blood and intra-osseous mesenchymal stromal cells, IO UCB + IV MSC = intra-osseous umbilical cord blood and INTRAVENOUS mesenchymal stromal cells $(*=\mathrm{p}<0.05 ; * *=\mathrm{p}<0.001)$. p-values as compared to the intravenous umbilical cord blood group.

Table 1. Summary of the percent CD45, CD3, CD13, CD14, CD19 in the right tibia bone marrow at 6 weeks post umbilical cord blood infusion. IV UCB $=$ intravenous umbilical cord blood, IO UCB = intra-osseous umbilical cord blood, IV UCB + IV MSC = intravenous umbilical cord blood and mesenchymal stromal cells, IO UCB + IO MSC $=$ intra-osseous umbilical cord blood and mesenchymal stromal cells, IV UCB + IO MSC = intravenous umbilical cord blood and intra-osseous mesenchymal stromal cells, IO UCB + IV MSC = intra-osseous umbilical cord blood and INTRAVENOUS mesenchymal stromal cells. p-values as compared to the intravenous umbilical cord blood group.

\begin{tabular}{|c|c|c|c|c|c|c|}
\hline & IV UCB & IO UCB & IV UCB + IO MSC & IV UCB + IV MSC & IO UCB + IV MSC & IO UCB + IO MSC \\
\hline CD 45 & $2.9 \%$ & $2.5 \%(p>0.05)$ & $7 \%(p>0.05)$ & $22 \%(p<0.01)$ & $31 \%(p<0.01)$ \\
\hline CD3 & $0.1 \%$ & $0.2 \%(p>0.05)$ & $0.2 \%(p>0.05)$ & $0.5 \%(p>0.05)$ & $1.4 \%(p>0.05)$ \\
\hline CD13 & $0.3 \%$ & $0.7 \%(p>0.05)$ & $2.6 \%(p>0.05)$ & $0.3 \%(p>0.05)$ & $4.8 \%(p>0.05)$ \\
\hline CD14 & $0.4 \%$ & $0.2 \%(p>0.05)$ & $0.3 \%(p>0.05)$ & $0.5 \%(p>0.05)$ & $0.01 \%(p<0.05)$ \\
\hline CD19 & $2.0 \%$ & $0.9 \%(p>0.05)$ & $4 \%(p>0.05)$ & $21 \%(p<0.01)$ & $0.9 \%(p>0.05)$ & $3 \%(p>0.05)$ \\
\hline
\end{tabular}

transplantation cohort compared to IV UCB only cohort (B-cell $28 \%$ vs. $2 \%$; T-cell $3 \%$ vs. $0.1 \%$; Figure 1 and Table 1 ). Both the IV UCB/ IV MSC and the IO UCB/IV MSC cohorts demonstrated significant improvement in engraftment over the IV UCB control. Specifically, $\mathrm{CD} 19^{+} \mathrm{B}$ cell recovery was significantly elevated in mice that received both UCB and MSC concurrently using the same route (IO or IV) of administration ( $28 \%$ vs. $21 \%$, respectively) as compared to $\mathrm{CD}_{1} 9^{+}$ B cell recovery when UCS and MSC were administered via different routes (IV/IO, 4\%, IO/IV, 3\%, respectively).

\section{Direct histologic evaluation of IO injected bone marrow}

Next, we correlated our flow cytometric analyses of hematopoietic recovery with direct cellularity assessment of the injected mouse bone marrow compartments (Figure 2). An analysis of the marrow cellularity by an un-biased hematopathologist revealed a myeloidto-erythroid ratio of roughly 10:1 in all the mouse cohorts (Table 2). Interestingly, although statistically non-significant, the number of megakaryocyte per high-powered field was highest in the cohort with IV co-infused UCB and MSC. The overall bone marrow cellularity varied widely between cohort samples, and no significant differences were detected among cohorts (Table 2). These morphological data did not directly correlate with the numerical analysis of hematopoietic and immune reconstitution within the bone marrow samples by the more quantitative flow cytometry analysis.

\section{Discussion}

In our current study, we demonstrated that IO co-transplantation of CD34-selected UCB and MSCs improved human hematopoietic cell engraftment over UCB controls administered IV. Interestingly, we also observed a predominance of early B-cell engraftment (CD19) within the bone marrow. Our observation is in agreement with previous published reports of human UCB engraftment in NOD/SCID mice $[21,22]$. It is not surprising that co-localizing MSCs and UCB within the bone improves engraftment, as MSCs support hematopoiesis through a number of mechanisms within the bone marrow niche. These mechanisms include cytokine support [16,17], especially within the perivascular space within the marrow where MSCs secrete CXCL12 and stem cell factor [18]. Furthermore, the injected MSC may have a role in replacing damaged or ineffective marrow resident MSCs. Our data demonstrating that IO co-administration of UCB and MSC resulted in a significant increase in both the $\mathrm{CD} 19^{+} \mathrm{B}$-cell and $\mathrm{CD}^{+} \mathrm{T}$-cell populations within the bone marrow suggest that MSCs, 
Table 2. Tabulation of histologic analysis from murine cohorts. IV UCB $=$ intravenous umbilical cord blood, IO UCB = intra-osseous umbilical cord blood, IV UCB + IV MSC = intravenous umbilical cord blood and mesenchymal stromal cells, IO UCB + IO MSC = intra-osseous umbilical cord blood and mesenchymal stromal cells, IV UCB + IO MSC $=$ intravenous umbilical cord blood and intra-osseous mesenchymal stromal cells, IO UCB + IV MSC = intra-osseous umbilical cord blood and intravenous mesenchymal stromal cells

\begin{tabular}{|c|c|c|c|c|c|c|}
\hline & IV UCB & IO UCB & IV UCB + IO MSC & IV UCB + IV MSC & IO UCB + IV MSC & IO UCB + IO MSC \\
\hline Megakaryocyte per high power field & 1.2 & 1.3 & 0.9 & 2.3 & 2.2 & 0.4 \\
\hline Myeloid -to-erythroid Ratio & $10: 1$ & $10: 1$ & $10: 1$ & $10: 1$ & $10: 1$ & $10: 1$ \\
\hline Cellularity & $62 \%$ & $68 \%$ & $82 \%$ & $50 \%$ & $46 \%$ & $47 \%$ \\
\hline
\end{tabular}
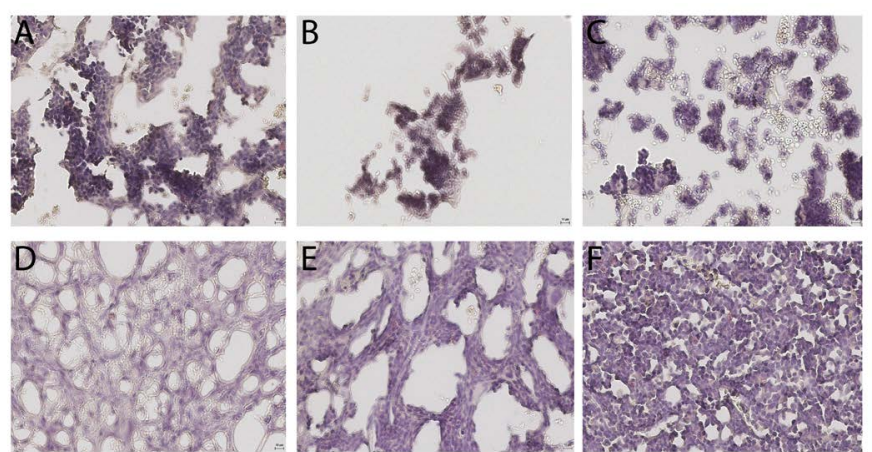

Figure 2. Histology of right tibia bone marrow from murine cohorts. A = intravenous (IV) umbilical cord blood (UCB), B = IV UBC and IV Mesenchymal Stromal Cells (MSC), C $=$ intra-osseous (IO) UCB, D = IO UCB and IO MSC, F = IO UCB and IV MSC, and F = IV UCB and IO MSC.

especially when directly co-infused into the bone marrow niche with UCBs, allow for a more robust immunologic re-constitution of the HSCs from UCB. Potential mechanisms by which MSC accomplish this is to act through both soluble factors, such as cytokines and growth factors, as well as via direct cell-cell contact with HSCs and cells of the bone marrow niche [23,24]. Direct contact of HSC by MSC in ex vivo expansion studies have demonstrated an increase in early lymphoid progenitors production [25,26]. Indeed, the importance of MSC support and interaction with the hematopoietic stem cell may be exemplified by the similar engraftment rate of the IO UCB cohort compared to the IV UCB cohort. The specific interaction between MSC and UCB in the bone marrow is not addressed in our study, and future investigations in this area may yield further scientific and mechanistic insights.

We did not observe a significant difference in $\mathrm{CD}_{4} 5^{+}, \mathrm{CD}^{+}, \mathrm{CD} 13^{+}$, $\mathrm{CD} 14^{+}$or $\mathrm{CD} 19^{+}$cells in the peripheral blood among any of our cohorts at week 3 and week 6 . However, human bone marrow engraftment in the absence of peripheral engraftment is considered an adequate surrogate marker in mice models [22].

Interestingly, a detailed histological analysis of the injected bone marrow did not demonstrate any differences in overall cellularity, megakaryocytic: erythrocytic (M:E) ratio, or the percentages of megakaryocytes among cohorts. This may be due to autologous recovery of murine bone marrow cells, which accompanied human UCB HSC engraftment. It is noteworthy that in the histologic evaluation of the IO MSC and IO UCB cohort, we observed an abundance of stromal tissue within the bone marrow microenvironment (Figure 2).

Overall, our results support and form the scientific basis for a new method of UCB transplantation that can be translated into clinical practice. UCB represents an important graft source for roughly one third of potential SCT patients who do not have a HLA matched related or unrelated donor $[27,28]$. However, UCB as a HSC source has its limitations, including lower cells dose resulting in slow or poor engraftment $[1,29,30]$. Various methods to optimize clinical UCB engraftment have been tried including ex-vivo expansion of UCB, intra- osseous transplantation of UCB, and intravenous co-transplantation of UCB and MSCs [12,20,31]. In addition, it has been demonstrated in patients that direct IO transplantation of a single UCB unit is safe, effective, and well tolerated following non-myeloablative conditioning preparatory regimen $[31,32]$. As a direct translation of our study presented here, we have now opened a clinical trial utilizing direct IO co-infusion of UCB and allogeneic MSC at the Seidman Cancer Center of the University Hospitals Case Medical Center (NCT02181478).

\section{Acknowledgements}

We thank Jane Reese and the Cellular Therapy Integrated Service of the National Center for Regenerative Medicine at Case Western Reserve University for their assistance.

\section{Funding}

This study was supported by NIH K12CA076917 Paul Calabresi Career Development Award for Clinical Oncology (K12): Clinical Oncology Research Career Development Program (L.M.III), Theresia G. \& Stuart F. Kline Family Foundation in Pediatric Oncology (A.Y.H.), and the Stem Cell Transplant Program at the Seidman Cancer Center at University Hospitals Case Medical Center.

\section{Author contributions}

Leland Metheny III: Concept and Design (L.M.III, M.d.L., A.Y.H), Collection and/or assembly of data (L.M.III, S.E., K.L., J.R., A.T., H.M.), Data analysis and interpretation (L.M.III, M.d.L., A.Y.H), Manuscript writing (L.M.III, M.d.L., A.Y.H), Final manuscript approval (L.M.III, M.d.L., A.Y.H.). Co-senior authors (A.Y.H., M.d.L.).

\section{Disclaimer}

The authors declare no conflict of interest.

\section{References}

1. Rocha V, Labopin M, Sanz G, Arcese W, Schwerdtfeger R, et al. (2004) Transplants of umbilical-cord blood or bone marrow from unrelated donors in adults with acute leukemia. N Engl J Med 351: 2276-2285. [Crossref]

2. Hogan CJ, Shpall EJ, McNulty O, McNiece I, Dick JE, et al. (1997) Engraftment and development of human CD34(+)-enriched cells from umbilical cord blood in NOD/ LtSz-scid/scid mice. Blood 90: 85-96. [Crossref]

3. Li ZY, Wang CQ, Lu G, Pan XY, Xu KL (2014) Effects of bone marrow mesenchymal stem cells on hematopoietic recovery and acute graft-versus-host disease in murine allogeneic umbilical cord blood transplantation model. Cell Biochem Biophys 70: 115-122.

4. Piacibello W, Sanavio F, Severino A, Danè A, Gammaitoni L, et al. (1999) Engraftment in nonobese diabetic severe combined immunodeficient mice of human CD34 $(+)$ cord blood cells after ex vivo expansion: evidence for the amplification and self-renewal of repopulating stem cells. Blood 93: 3736-3749. [Crossref]

5. Wang J, Kimura T, Asada R, Harada S, Yokota S, et al. (2003) SCID-repopulating cell activity of human cord blood-derived CD34- cells assured by intra-bone marrow injection. Blood 101: 2924-2931. [Crossref]

6. Cui J, Wahl RL, Shen T (1999) Bone marrow cell trafficking following intravenous administration. Br J Haematol 107: 895-902.

7. Van Hennik PB, de Koning AE, Ploemacher RE (1999) Seeding efficiency of primitive 
human hematopoietic cells in nonobese diabetic/severe combined immune deficiency mice: implications for stem cell frequency assessment. Blood 94: 3055-3061. [Crossref]

8. Zhong JF, Zhan Y, Anderson WF, Zhao Y (2002) Murine hematopoietic stem cell distribution and proliferation in ablated and nonablated bone marrow transplantation. Blood 100: 3521-3526. [Crossref]

9. Castello S, Podesta M, Menditto VG (2004) Intra-bone marrow injection of bone marrow and cord blood cells: an alternative way of transplantation associated with a higher seeding efficiency. Exp Hematol 32: 782-787.

10. Mazurier F, Doedens M, Gan OI, Dick JE (2003) Rapid myeloerythroid repopulation after intrafemoral transplantation of NOD-SCID mice reveals a new class of human stem cells. Nat Med 9: 959-963.

11. Robinson SN, Ng J, Niu T, Yang H, McMannis JD, et al. (2006) Superior ex vivo cord blood expansion following co-culture with bone marrow-derived mesenchymal stem cells. Bone Marrow Transplant 37: 359-366. [Crossref]

12. De Lima M, McNiece I, Robinson SN, Munsell M, Eapen M, et al. (2012) Cord-blood engraftment with ex vivo mesenchymal-cell coculture. $N$ Engl J Med 367: 2305-2315. [Crossref]

13. Verfaillie CM (1992) Direct contact between human primitive hematopoietic progenitors and bone marrow stroma is not required for long-term in vitro hematopoiesis. Blood 79: 2821-2826.

14. Choudhury C, Sparks R (1989) Role of the microenvironment on hematopoiesis. II. Regulation of cell kinetics in vitro during granulopoiesis and megakaryocytopoiesis. The Journal of Laboratory and Clinical Medicine 114: 382-388.

15. Carrancio S, Blanco B, Romo C, Muntion S, Lopez-Holgado N, et al. (2011) Bone marrow mesenchymal stem cells for improving hematopoietic function: an in vitro and in vivo model. Part 2: Effect on bone marrow microenvironment. PLoS One 6: e26241. [Crossref]

16. Cheng L, Qasba P, Vanguri P, Thiede MA (2000) Human mesenchymal stem cells support megakaryocyte and pro-platelet formation from $\mathrm{CD} 34(+)$ hematopoietic progenitor cells. J Cell Physiol 184: 58-69. [Crossref]

17. Majumdar MK, Thiede MA, Mosca JD, Moorman M, Gerson SL (1998) Phenotypic and functional comparison of cultures of marrow-derived mesenchymal stem cells (MSCs) and stromal cells. Journal of Cellular Physiology. Jul 1998;176(1):57-66.

18. Méndez-Ferrer S, Michurina TV, Ferraro F, Mazloom AR, Macarthur BD, et al. (2010) Mesenchymal and haematopoietic stem cells form a unique bone marrow niche. Nature 466: 829-834. [Crossref]

19. Bensidhoum M, Chapel A, Francois S, Demarquay C, Mazurier C, et al. (2004) Homing of in vitro expanded Stro-1- or Stro-1+ human mesenchymal stem cells into the NOD/ SCID mouse and their role in supporting human CD34 cell engraftment. Blood 103: 3313-3319. [Crossref]
20. Macmillan ML, Blazar BR, DeFor TE, Wagner JE (2009) Transplantation of exvivo culture-expanded parental haploidentical mesenchymal stem cells to promote engraftment in pediatric recipients of unrelated donor umbilical cord blood: results of a phase I-II clinical trial. Bone Marrow Transplant 43: 447-454. [Crossref]

21. Carrancio S, Romo C, Ramos T, Lopez-Holgado N, Muntion S, et al. (2013) Effects of MSC coadministration and route of delivery on cord blood hematopoietic stem cell engraftment. Cell Transplant 22: 1171-1183. [Crossref]

22. Ueda T, Yoshino H, Kobayashi K, Kawahata M, Ebihara Y, et al. (2000) Hematopoietic repopulating ability of cord blood CD34(+) cells in NOD/Shi-scid mice. Stem Cells 18 204-213. [Crossref]

23. Oostendorp RA, Robin C, Steinhoff C, Marz S, Bräuer R, et al. (2005) Long-term maintenance of hematopoietic stem cells does not require contact with embryo-derived stromal cells in cocultures. Stem Cells 23: 842-851. [Crossref]

24. Wagner W, Wein F, Roderburg C (2007) Adhesion of hematopoietic progenitor cells to human mesenchymal stem cells as a model for cell-cell interaction. Exp Hematol 35 : 314-325. [Crossref]

25. Da Silva CL, Goncalves R, dos Santos F, Andrade PZ, Almeida-Porada G, et al. (2010) Dynamic cell-cell interactions between cord blood haematopoietic progenitors and the cellular niche are essential for the expansion of CD34+, CD34+CD38- and early lymphoid CD7+ cells. J Tissue Eng Regen Med 4: 149-158. [Crossref]

26. Frias AM, Porada CD, Crapnell KB, Cabral JM, Zanjani ED, et al. (2008) Generation of functional natural killer and dendritic cells in a human stromal-based serum-free culture system designed for cord blood expansion. Exp Hematol 36: 61-68. [Crossref]

27. Karanes C, Nelson GO, Chitphakdithai P (2008) Twenty years of unrelated donor hematopoietic cell transplantation for adult recipients facilitated by the National Marrow Donor Program. Biology of Blood and Marrow Transplantation 14: 8-15.

28. Confer D, Robinett P (2008) The US National Marrow Donor Program role in unrelated donor hematopoietic cell transplantation. Bone Marrow Transplant 42 Suppl 1: S33S5. [Crossref]

29. Laughlin MJ, Barker J, Bambach B, Koc ON, Rizzieri DA, et al. (2001) Hematopoietic engraftment and survival in adult recipients of umbilical-cord blood from unrelated donors. $N$ Engl J Med 344: 1815-1822. [Crossref]

30. Cornetta K, Laughlin M, Carter S (2005) Umbilical cord blood transplantation in adults: results of the prospective Cord Blood Transplantation (COBLT). Biol Blood Marrow Transplant 11: 149-160.

31. Frassoni F, Gualandi F, Podestà M, Raiola AM, Ibatici A, et al. (2008) Direct intrabone transplant of unrelated cord-blood cells in acute leukaemia: a phase I/II study. Lancet Oncol 9: 831-839. [Crossref]

32. Rocha V, Labopin M, Ruggeri A (2013) Unrelated cord blood transplantation: outcomes after single-unit intrabone injection compared with double-unit intravenous injection in patients with hematological malignancies. Transplantation 95: 1284-1291.

Copyright: (C2016 Metheny L. This is an open-access article distributed under the terms of the Creative Commons Attribution License, which permits unrestricted use, distribution, and reproduction in any medium, provided the original author and source are credited. 